\title{
Inteligencia emocional en las víctimas de acoso escolar y en los agresores
}

\author{
Maite Garaigordobil ${ }^{1}$ y José A. Oñederra ${ }^{2}$ \\ ${ }^{1}$ Universidad del País Vasco, ${ }^{2}$ Instituto de Bidebieta, San Sebastián (España)
}

\begin{abstract}
El estudio tuvo como principal objetivo analizar las relaciones existentes entre ser víctima de acoso escolar y ser agresor con parámetros asociados a la inteligencia emocional (emotividad, eficacia, pensamiento supersticioso, rigidez, pensamiento esotérico, ilusión). La muestra está constituida por 248 alumnos de 12 a 16 años, 144 varones (58.1\%) y 104 mujeres (41.9\%). Para medir las variables se utilizaron 3 instrumentos de evaluación: el Inventario de Pensamiento Constructivo, la Lista de Chequeo mi vida en la escuela y el Cuestionario de Conductas Antisociales-Delictivas. Los resultados obtenidos confirmaron que: 1) los adolescentes que habían sufrido muchas conductas de intimidación o bullying, tuvieron bajo nivel de inteligencia emocional, baja emotividad, baja autoestima, baja tolerancia a la frustración, baja eficacia, y poca actividad; y 2) Los adolescentes que tenían un nivel alto de conductas antisociales-delictivas mostraban bajo nivel de inteligencia emocional, de eficacia, de actividad, de responsabilidad, y de tolerancia. La discusión gira en torno a la importancia de implementar programas para fomentar la inteligencia emocional con la finalidad de prevenir el acoso escolar.
\end{abstract}

Palabras clave: Acoso escolar, inteligencia emocional, conducta antisocial, género.

Emotional intelligence in victims of school bullying and in aggressors. The purpose of this study was to analyze the relations between being the victim of school bullying and being the aggressor with the parameters associated with emotional intelligence (emotionality, efficacy, superstitious thinking, rigidity, esoteric thinking, illusion). The sample is made up of 248 students from 12 to 16 years of age, 144 male (58.1\%) and 104 female (41.9\%). To measure the variables, three assessment instruments were used: the Constructive Thinking Inventory, the My Life at School Checklist and the Antisocial-Delinquent Behavior Questionnaire. The results obtained confirmed that: 1) adolescents who had suffered many behaviors of intimidation or bullying, had a low level of emotional intelligence, low emotionality, low self-esteem, low frustration tolerance, low efficacy, and little activity; 2) the adolescents who had a high level of antisocial-delinquent behaviors showed a low level of emotional intelligence, low efficacy, low activity, low responsibility, and low tolerance. The discussion addresses the importance of implementing programs to promote emotional intelligence in order to prevent bullying at school.

Key words: School bullying, emotional intelligence, antisocial behavior, gender.

Correspondencia: Maite Garaigordobil. Universidad del País Vasco. Facultad de Psicología. Avda. de Tolosa 70. C.P. 20018. Donostia-San Sebastián (España). E-mail: maite.garaigordobil@ehu.es 
El término "Inteligencia Emocional" (IE) se refiere a la capacidad de reconocer los propios sentimientos y los ajenos, de motivarse y manejar bien las emociones tanto en relación a uno mismo como a los demás, y describe aptitudes complementarias, pero distintas de la inteligencia académica, las habilidades puramente cognitivas medidas por el CI (cociente intelectual). La importancia de la IE radica en aprender a administrar las emociones para que éstas trabajen a favor de la persona. La investigación ha evidenciado que la carencia de IE, incide directamente en el rendimiento escolar, además de mostrar un desempeño más pobre en muchos aspectos (problemas sociales, ansiedad, depresión, agresividad...). La carencia de alfabetización en IE en las instituciones educativas de nuestro medio ha incidido en el comportamiento aislado, deprimido, indisciplinado, impulsivo y agresivo de los estudiantes y también en sus aprendizajes pedagógicos. El bajo desarrollo de la IE acentúa el aislamiento, la ansiedad, la depresión, los problemas de atención o del pensamiento, la delincuencia y la agresividad (Goleman, 1996). En esta dirección, Lopes, Salovey y Straus (2003) y Ciarrochi, Chan y Bajgar (2001) constatan que alumnos con puntuaciones altas en IE tienen una mayor satisfacción en las relaciones con sus amigos, interacciones más positivas y menos conflictos con ellos. Asimismo, León (2009) enfatiza que un déficit en la IE provoca y facilita la aparición de problemas conductuales en el ámbito de las relaciones interpersonales, y afecta al bienestar psicológico.

Todos los estudios que han analizado la inteligencia emocional en las víctimas de bullying han evidenciado que la víctima es la parte que más sufre las consecuencias del acoso escolar en todas las facetas. Hoover, Oliver y Hazler (1992) hallaron que los alumnos identificados como víctimas autoevaluaban los efectos del acoso que sufrían como severos en las dimensiones del autoconcepto social, emocional, académica, somática y familiar. Muchos estudios han puesto de relieve que las víctimas muestran baja autoestima, bajo autoconcepto (Eslea et al., 2004; Estévez, Martínez y Musitu, 2006; Mynard y Joseph, 1997; Nansel et al., 2001; O’Moore, 1997; Olweus, 1993; Piñuel y Oñate, 2006; Slee y Rigby, 1993; Van Der Meulen et al., 2003). Estudios que han diferenciado entre víctimas pasivas y víctimas agresoras, confirman que las primeras tienen baja autoestima y las segundas alta como los agresores dominantes (Hanish y Guerra, 2004), y otros estudios han mostrado bajo autoconcepto emocional y social en las víctimas (de la Torre, García, Carpio y Casanova, 2008).

En las últimas décadas son muchos los estudios que han explorado diversas variables relacionadas con la inteligencia emocional en los bullies o agresores. Los estudios que han analizado la autoestima en los agresores han puesto de relieve resultados contradictorios. Algunos han mostrado que los que intimidan tienen un nivel alto de autoestima (Batsche y Knoff, 1994; Díaz-Aguado, 2004; Estévez et al., 2006; Olweus, 1993), otros han evidenciado que los agresores tienen como mínimo niveles medios de autoestima (Parkhurst y Asher, 1992), mientras que otros han hallado un nivel 
bajo (Esteve, Merino y Cantos, 2001; O’Moore, 1997) y una autoestima más negativa en las dimensiones familiar y escolar (Estévez, Herrero, Martínez y Musitu, 2006). Algunos estudios que diferencian entre agresores dominantes y ansiosos, evidencian que los primeros presentan alta autoestima (Hanish y Guerra, 2004) y los segundos baja (Cammack-Barry, 2005). En el contexto de esta controversia Baumeister (2001) concluye que el nivel de autoestima nada tiene que ver con la conducta agresiva.

Por otro lado, Liau, Liau, Teoh y Liau (2003) constatan que los alumnos de secundaria con inteligencia emocional más baja, muestran mayores niveles de conductas agresivas y delictivas; y en la misma dirección, Zimmerman (2005) concluye que los agresores tienen un bajo índice de inteligencia emocional, concretamente en los aspectos de empatía, autocontrol y habilidades sociales. Caspi et al. (1994) asociaron la conducta delictiva de los adolescentes a un débil autocontrol y a una emotividad negativa (tendencia a estar enojado, ansioso e irritable). Zuckerman (1994) estudiando el constructo de búsqueda de sensaciones constató su relación con la carencia de respeto a las normas sociales, la baja responsabilidad y el bajo autocontrol. Contreras y García (2008) evidencian en niños de 3 a 5 años la vinculación del razonamieno contrafáctico con aspectos de la inteligencia cognitiva, social y emocional, en los que el agresor tiene deficiencias. Además, otros estudios han puesto de relieve que el estudiante violento muestra deficiencias en dos cualidades esenciales de la inteligencia emocional, en autocontrol y empatía (Avilés y Monjas, 2005; Díaz-Aguado, 2006; Henley y Long, 1999; Hernández, Sarabia y Casares, 2002; Pelegrín y Garcés de Los Fayos, 2008; Stassen, 2007), evidenciando la importancia de la empatía como elemento favorecer de la convivencia (Martorell, González, Rasal y Estellés, 2009). En este sentido, Rubin (1999) observó que los alumnos de primaria que obtenían mayores puntuaciones en inteligencia emocional eran evaluados por sus compañeros como menos agresivos y sus profesores los consideraban más propensos a los comportamientos prosociales. Una revisión del bullying, en relación a su epidemiología, prevención e intervención, puede consultarse en un trabajo reciente (Garaigordobil y Oñederra, 2010).

Tomando como base las investigaciones previas, el presente estudio tuvo como principal objetivo analizar las relaciones existentes entre ser víctima de acoso escolar y ser agresor con diversos parámetros asociados a la inteligencia emocional o pensamiento constructivo global (emotividad, eficacia, pensamiento supersticioso, rigidez, pensamiento esotérico e ilusión). En este contexto se proponen 4 hipótesis: 1) Los adolescentes que reciban muchas conductas sociales positivas por parte de sus compañeros, tendrán alto pensamiento constructivo global o inteligencia emocional, alto nivel de pensamiento positivo y de responsabilidad, serán poco suspicaces, y tendrán pocas creencias irracionales; 2) Los adolescentes que reciban muchas conductas de intimidación o bullying de sus compañeros, así como aquellos que tengan un alto índice de agresión, tendrán bajo pensamiento constructivo global o inteligencia emocional, baja 
emotividad, baja autoestima, baja tolerancia a la frustración, baja eficacia, y poca actividad; 3) Los adolescentes que tengan un nivel alto de conductas antisocialesdelictivas mostrarán bajo nivel de pensamiento constructivo global o inteligencia emocional, bajo nivel de eficacia, de actividad, de responsabilidad, y alto nivel de intolerancia; y 4) No se encontrarán diferencias significativas en las variables exploradas, es decir, en recepción de conductas sociales, realización de conductas antisociales-delictivas e inteligencia emocional, en función del estatus o nivel socioeconómico-cultural.

\section{METODO}

\section{Participantes}

La muestra está constituida por 248 alumnos de Educación Secundaria Obligatoria (ESO), de 12 a 16 años, pertenecientes en 3 institutos de la ciudad de Donostia-San Sebastián. Del conjunto de la muestra 144 son varones (58.1\%) y 104 mujeres (41.9\%). Los participantes cursan los 4 niveles educativos de ESO ( $1^{\circ} \mathrm{ESO}$ $N=69,27.8 \%$; $2^{\circ}$ ESO: $N=68,27.4 \% ; 3^{\circ}$ ESO: $N=55,22.2 \% ; 4^{\circ}$ ESO: $N=56$, $22.6 \%$ ). El criterio para la selección muestral fue elegir centros de distintos niveles socioeconómicos y culturales: 1) clase alta y media-alta y, culturalmente bien formados $(N=100,40.3 \%) ; 2)$ clase media y, con una formación cultural diversa $(N=75,30.2 \%)$; 3) familias humildes y de clase baja con escasa formación cultural y un alto índice de inmigrantes $(N=73,29.4 \%)$. Se seleccionó de forma aleatoria un centro en cada estatus. La población total de alumnos de ESO de Donostia-San Sebastián es de 7.106, 2.039 pertenecen a la red pública y 5.067 a la privada. Por lo tanto, la muestra de 248 alumnos representa el $3.5 \%$.

\section{Instrumentos}

Para evaluar las variables objeto de estudio se utilizaron 3 instrumentos de evaluación con adecuadas garantías psicométricas de fiabilidad y validez.

CTI. Inventario de pensamiento constructivo. Una medida de la inteligencia emocional (Epstein, 2001). Compuesto por 108 ítems que evalúan el pensamiento constructivo y la inteligencia emocional. El instrumento está jerárquicamente organizado y ofrece información en tres niveles de generalidad. El nivel más general consiste en una escala global que se denomina PCG (Pensamiento Constructivo Global). Las personas con altas puntuaciones en PCG tienen una estructura de pensamiento flexible, adaptan sus modos de pensar a las modalidades de diferentes situaciones, se aceptan bien a sí mismas y a los demás, suelen establecer relaciones gratificantes y generalmente tienden a conceder a otros el beneficio de la duda. El siguiente nivel de generalidad lo componen 6 escalas principales que miden las formas básicas de pensamiento constructivo o destructivo que a su vez contienen 15 subescalas: 1) Emotividad: autoestima, tolerancia 
a la frustración, impasibilidad, capacidad de sobreponerse; 2) Eficacia: pensamiento positivo, actividad, responsabilidad; 3) Pensamiento supersticioso; 4) Rigidez: extremismo, suspicacia, intolerancia; 5) Pensamiento esotérico: creencias paranormales, creencias irracionales; y 6) Ilusión: euforia, pensamiento estereotipado, ingenuidad. La validez del CTI se apoya en una gran variedad de estudios que han analizado las relaciones de las escalas con tests de personalidad, conducta observada, tests de inteligencia, éxito en el trabajo, bienestar emocional-físico...

LC. Lista de chequeo: mi vida en la escuela (Arora, 1987). Describe un conjunto de conductas realizadas por otros alumnos hacia el propio alumno evaluado que se convierte en víctima en el caso de padecer agresión o intimidación. Se configura con 39 afirmaciones con las que se mide el índice de bullying y el índice general de agresión. Los adolescentes informan con qué frecuencia (nunca, una vez, más de una vez) durante esa semana les ha pasado lo que dicen las frases en el centro educativo. Los ítems que informan del bullying y del índice general de agresión son: intentó darme patadas, me dijeron que me darían una paliza, intentó que le diera dinero, intentó hacerme daño, trataron de romperme algo mío, intentaron pegarme. A partir de ellos se calcula el índice de bullying (más de una vez) y el índice general de agresión (una vez y más de una vez) que es más amplio que el índice bullying porque incluye conductas más ocasionales. Además, en este estudio se miden 2 tipos de conductas que el adolescente dice haber recibido de sus compañeros: las conductas positivas (14 afirmaciones: me ha dicho algo bonito, fue muy amable conmigo, me ha sonreído, me ayudó con mi trabajo, jugó conmigo...), y las conductas negativas (19 afirmaciones: me ha dicho motes, gente intentó hacerme daño, me hizo hacer algo que no quería hacer, me ha quitado alguna cosa, me gritó, se rió de mí horriblemente...). Para la corrección se asigna 1 punto a cada ítem señalado "una vez" y 2 puntos en "más de una vez"; y se obtiene la suma de los puntos en las 4 dimensiones evaluadas. En un estudio psicométrico (Garaigordobil, 2009), con adolescentes de 15 a 17 años, se confirmó la consistencia interna de LC en las 4 dimensiones (alpha de Cronbach entre .84 y .85), así como su fiabilidad test-retest (correlaciones entre .64 y .69).

AD. Cuestionario de conductas antisociales-delictivas (Seisdedos, 1988/1995). Con 40 frases evalúa 2 aspectos de la conducta desviada, el antisocial (entrar a un sitio prohibido, hacer pintadas, romper o tirar al suelo cosas de otra persona, pelearse con otros, con golpes, insultos o palabras ofensivas...), y el delictivo (tomar drogas, destrozar o dañar lugares públicos, robar, conseguir dinero amenazando a personas más débiles, pertenecer a una pandilla que se mete en peleas o crea disturbios...). La tarea consiste en informar si se han realizado las conductas que se indican en las frases, y se otorga 1 punto por cada respuesta afirmativa. Los coeficientes de Spearman-Brown (.86 en ambas escalas) confirman la fiabilidad del AD. La validez 
criterial se basó en las puntuaciones significativamente superiores obtenidas por 95 adolescentes con problemas de conducta en contraste con 99 adolescentes de control.

\section{Procedimiento}

El estudio utilizó una metodología descriptiva y correlacional de corte transversal buscando establecer relaciones de concomitancia entre el rol de víctima y agresor con la inteligencia emocional (pensamiento constructivo global) y factores asociados a la misma (emotividad, eficacia, pensamiento supersticioso, rigidez, pensamiento esotérico, e ilusión). Después de presentar el proyecto en los centros educativos, se solicitó el consentimiento informado, y se llevó a cabo la aplicación de los instrumentos en una sesión de evaluación que fue realizada por un Licenciado en Psicología.

\section{RESULTADOS}

Índice de bullying en las víctimas y conducta antisocial-delictiva en los agresores: diferencias en función del sexo

Después de realizar la prueba de Levene para verificar el supuesto de homocedasticidad encontrando igualdad de varianzas, se calcularon las medias, desviaciones típicas, y se realizó un análisis de varianza (ANOVA) para la recepción de conductas sociales diversas por parte de los compañeros y para la conducta antisocialdelictiva en función del sexo.

Tabla 1. Medias, desviaciones típicas y resultados del análisis de varianza en función del sexo en recepción de conductas sociales y realización de conductas antisociales-delictivas

\begin{tabular}{lccccc}
\hline & \multicolumn{3}{c}{ Chicos } & \multicolumn{2}{c}{ Chicas } \\
\cline { 2 - 5 } & $M$ & $D T$ & $M$ & $D T$ & $(1,246)$ \\
\hline LC. Conductas positivas & 9.23 & 3.25 & 10.22 & 2.64 & $6.56 *$ \\
LC. Conductas negativas & 4.82 & 3.89 & 4.15 & 3.75 & 1.83 \\
LC. Índice de Bullying & 0.24 & 0.72 & 0.13 & 0.41 & 2.20 \\
LC. Índice general de agresión & 0.78 & 1.32 & 0.52 & 1.23 & 2.48 \\
\hline AD. Conducta antisocial & 6.85 & 5.12 & 7.26 & 4.89 & 0.39 \\
AD. Conducta delictiva & 1.64 & 3.44 & 0.93 & 1.92 & 3.58 \\
AD. Conducta antisocial-delictiva & 8.49 & 7.37 & 8.19 & 6.17 & 0.11 \\
\hline$* p<.05$ & & & & &
\end{tabular}

Los resultados (Tabla 1) muestran que apenas existen diferencias significativas entre chicos y chicas, ya que tienen un índice de bullying, un índice general de agresión, y un nivel de conducta antisocial-delictiva similar. Por lo tanto, los coeficientes de Pearson se obtienen con el conjunto de la muestra.

Inteligencia emocional en las víctimas de acoso escolar

Con la finalidad de analizar las relaciones existentes entre recibir diversos tipos de conductas por parte de los compañeros y la inteligencia emocional se obtuvieron los coeficientes de correlación de Pearson cuyos resultados se exponen en la tabla 2. 
Tabla 2. Correlaciones de Pearson entre conductas positivas, negativas, bullying e índice

\begin{tabular}{|c|c|c|c|c|}
\hline & $\begin{array}{c}\text { Conductas } \\
\text { positivas }\end{array}$ & $\begin{array}{l}\text { Conductas } \\
\text { negativas }\end{array}$ & $\begin{array}{c}\text { Índice } \\
\text { de bullying }\end{array}$ & $\begin{array}{c}\text { Índice } \\
\text { de agresión }\end{array}$ \\
\hline PENS. CONSTRUCTIVO GLOBAL & $.16 * *$ & $-.27 * * *$ & $-.22 * * *$ & $-.22 * * *$ \\
\hline EMOTIVIDAD & .02 & $-.23 * * *$ & $-.16 * *$ & $-.16 *$ \\
\hline Autoestima & .06 & $-.16 * *$ & $-.14 *$ & $-.12 *$ \\
\hline Tolerancia a la frustración & .02 & $-.23 * * *$ & $-.17 * *$ & $-.21 * * *$ \\
\hline Impasibilidad & -.04 & $-.18 * *$ & -.11 & -.06 \\
\hline Capacidad de sobreponerse & .02 & $-.12 *$ & -.07 & -.08 \\
\hline EFICACIA & .11 & $-.27 * * *$ & $-.20 * * *$ & $-.24 * * *$ \\
\hline Pensamiento positivo & $.18 * *$ & -.09 & -.06 & -.06 \\
\hline Actividad & .07 & $-.25 * * *$ & $-.21 * * *$ & $-.19 * *$ \\
\hline Responsabilidad & $.13 *$ & -.06 & $-.14 *$ & -.05 \\
\hline PENS. SUPERSTICIOSO & .11 & $.16 * *$ & .10 & $.17 * *$ \\
\hline RIGIDEZ & -.08 & $.15 *$ & .04 & .11 \\
\hline Extremismo & .01 & .08 & -.01 & .08 \\
\hline Suspicacia & $-.17 * *$ & $.18 * *$ & .07 & $.15 *$ \\
\hline Intolerancia & -.07 & .05 & .03 & .00 \\
\hline PENS. ESOTÉRICO & .04 & $.12 *$ & .09 & .11 \\
\hline Creencias paranormales & -.03 & .09 & .04 & .09 \\
\hline Creencias irracionales & $-.13 *$ & $.13 *$ & $.12 *$ & .10 \\
\hline ILUSIÓN & $.12 *$ & -.00 & -.01 & -.00 \\
\hline Euforia & .11 & .05 & .04 & .07 \\
\hline Pensamiento estereotipado & -.03 & -.00 & -.10 & -.01 \\
\hline Ingenuidad & $.17 * *$ & .01 & .02 & .05 \\
\hline
\end{tabular}

Como se puede observar en la tabla 2, en primer lugar, los coeficientes de correlación obtenidos entre el Pensamiento Constructivo Global (PCG), sugieren que los adolescentes que tenían alto nivel de PCG, es decir, alta inteligencia emocional, también recibían muchas conductas sociales positivas de sus compañeros $(r=.16)$, pocas conductas negativas $(r=-.27)$, pocas conductas de intimidación o bullying $(r=-.22)$, y mostraban un índice general de agresión bajo $(r=-.22)$.

En segundo lugar, los resultados evidencian que la emotividad no está relacionada con la recepción de conductas sociales positivas con los iguales. Sin embargo, se encontraron correlaciones significativas inversas $(p<.05)$ entre la recepción de conductas sociales negativas con emotividad $(r=-.23)$, y con todas las variables incluidas en esta dimensión (autoestima, tolerancia a la frustración, impasibilidad y capacidad de sobreponerse). En relación al índice de bullying y al índice general de agresión se evidenciaron correlaciones significativas inversas de ambos indicadores con emotividad ( $r=-.16$ respectivamente), y con dos variables incluidas en esta dimensión (autoestima y tolerancia a la frustración). En tercer lugar, no se encontraron relaciones entre eficacia y recepción de conductas positivas aunque si se hallaron relaciones directas con dos variables incluidas en esta dimensión (pensamiento positivo y responsabilidad). Se encontraron correlaciones inversas $(p<.01)$ de la recepción de conductas sociales negativas con eficacia $(r=-.27)$, y una variable de esta dimensión (actividad); del índice de bullying con eficacia $(r=-.20)$, y dos variables (actividad y 
responsabilidad), así como del índice general de agresión y eficacia $(r=-.24)$, y una de sus variables (actividad). En cuarto lugar, los resultados pusieron de relieve correlaciones significativas positivas $(p<.01)$ del pensamiento supersticioso con recepción de conductas negativas por parte de los iguales $(r=.16)$, y con el índice general de agresión $(r=.17)$.

En quinto lugar, en relación con la dimensión rigidez, se confirman correlaciones inversas $(p<.05)$ entre suspicacia y recepción de conductas sociales positivas $(r=-.17)$, así como correlaciones directas entre recepción de conductas sociales negativas con rigidez $(r=.15)$, y suspicacia $(r=.18)$, y también entre el índice general de agresión y suspicacia $(r=.15)$. En sexto lugar, se hallaron correlaciones directas $(p<.05)$ entre pensamiento esotérico y recepción de conductas sociales negativas $(r=.12)$. Además, se encontraron correlaciones inversas entre creencias irracionales y recepción de conductas sociales positivas $(r=-.13)$, así como correlaciones directas entre creencias irracionales y recepción de conductas negativas ( $r=.13)$ y de conductas de intimidación o bullying $(r=.12)$. Finalmente, la variable ilusión apenas tuvo correlaciones con las variables evaluadas. Únicamente se hallaron relaciones directas con recepción de conductas sociales positivas $(r=.12)$, y con ingenuidad $(r=.17)$ una de las variables de la dimensión ilusión.

En síntesis, los resultados obtenidos han puesto de relieve que: 1) los adolescentes que recibían muchas conductas sociales positivas de sus compañeros, tenían alto pensamiento constructivo global o inteligencia emocional, alto nivel de pensamiento positivo y de responsabilidad, eran poco suspicaces, tenían pocas creencias irracionales, alto nivel de ilusión y de ingenuidad; 2) Los adolescentes que recibían muchas conductas sociales negativas de sus compañeros, manifestaban bajo nivel de pensamiento constructivo global o inteligencia emocional, baja emotividad, baja autoestima, baja tolerancia a la frustración, baja impasibilidad, baja capacidad de sobreponerse, baja eficacia, poca actividad, alto nivel de pensamiento supersticioso, mucha rigidez, eran suspicaces, tenían altas puntuaciones en pensamiento esotérico y muchas creencias irracionales; 3) Los adolescentes que habían recibido muchas conductas de intimidación de sus compañeros (alto índice de bullying) tenían bajo nivel de pensamiento constructivo global o inteligencia emocional, baja emotividad, baja autoestima, baja tolerancia a la frustración, eran poco eficaces, tenían bajo nivel de actividad, de responsabilidad, y muchas creencias irracionales; y 4) Los adolescentes que tenían alto índice general de agresión, mostraban bajo pensamiento constructivo global o inteligencia emocional, baja emotividad, baja autoestima, baja tolerancia a la frustración, eran poco eficaces, tenían un nivel bajo de actividad, alto nivel de pensamiento supersticioso y de suspicacia. 


\section{Inteligencia emocional en los agresores}

Con la finalidad de analizar las relaciones existentes entre la conducta antisocial-delictiva y la inteligencia emocional se obtuvieron los coeficientes de correlación de Pearson cuyos resultados se exponen en la tabla 3.

Tabla 3. Correlaciones de Pearson entre conducta antisocial y delictiva con inteligencia emocional

\begin{tabular}{lccc}
\hline & Antisocial & Delictiva & Antisocial + Delictiva \\
\hline PENS. CONSTRUCTIVO GLOBAL & -.11 & $-.15 *$ & $-.15^{*}$ \\
\hline EMOTIVIDAD & -.07 & -.04 & -.07 \\
Autoestima & -.02 & $-.16 * *$ & -.08 \\
Tolerancia a la frustración & -.08 & .04 & -.08 \\
Impasibilidad & .02 & .05 & .00 \\
Capacidad de sobreponerse & -.08 & -.00 & -.06 \\
\hline EFICACIA & $-.15 *$ & $-.20 * * *$ & -.07 \\
Pensamiento positivo & -.01 & $-.15 *$ & $-.20^{* * *}$ \\
Actividad & $-.16^{* *}$ & $-.19 * *$ & $-.26^{* * *}$ \\
Responsabilidad & $-.23^{* * *}$ & $-.21 * * *$ & .00 \\
\hline PENS. SUPERSTICIOSO & -.00 & .00 & .01 \\
\hline RIGIDEZ & .02 & .01 & $-.15 *$ \\
Extremismo & $-.17 * *$ & -.06 & .00 \\
Suspicacia & .00 & .00 & .09 \\
Intolerancia & $.18 * *$ & .05 & .10 \\
\hline PENS. ESOTÉRICO & .07 & .10 & .03 \\
Creencias paranormales & .09 & .08 & $-.14 *$ \\
Creencias irracionales & .00 & .08 & -.06 \\
\hline ILUSIÓN & $-.15 *$ & -.07 & $.07 * *$ \\
Euforia & -.04 & .07 & .02 \\
Pensamiento estereotipado & $-.26 * * *$ & -.10 & \\
Ingenuidad & -.02 & -.01 &
\end{tabular}

Los resultados obtenidos (Tabla 3) evidenciaron correlaciones significativas negativas $(p<.05)$ entre la conducta antisocial-delictiva con diversas variables tales como pensamiento constructivo global $(r=-.15)$, eficacia $(r=-.19)$, actividad $(r=-.20)$, responsabilidad $(r=-.26)$, extremismo $(r=-.15)$, ilusión $(r=-.14)$, pensamiento estereotipado $(r=-.23)$ y correlaciones positivas con intolerancia $(r=.16)$. Estos datos sugieren que los adolescentes que realizaban muchas conductas antisociales-delictivas mostraban bajo nivel de pensamiento constructivo global o inteligencia emocional, bajo nivel de eficacia, de actividad, de responsabilidad, de extremismo, de ilusión, de pensamiento estereotipado y de tolerancia.

Recepción de conductas sociales, realización de conductas antisocialesdelictivas e inteligencia emocional: Diferencias en función del estatus socio-económicocultural

Con la finalidad de analizar si existen diferencias en función del estatus socioeconómico-cultural en las variables exploradas, se realizó un análisis de varianza cuyos resultados se presentan en la tabla 4 . 
Tabla 4. Medias, desviaciones típicas, y resultados del análisis de varianza en función del estatus socioeconómico-cultural para recepción de conductas sociales, realización de conductas antisociales-delictivas e inteligencia emocional

\begin{tabular}{|c|c|c|c|c|c|c|c|}
\hline & \multicolumn{2}{|c|}{ Bajo } & \multicolumn{2}{|c|}{ Medio } & \multicolumn{2}{|c|}{ Alto } & \multirow{2}{*}{$\begin{array}{c}F \\
(2,247)\end{array}$} \\
\hline & $M$ & $D T$ & $M$ & $D T$ & $M$ & $D T$ & \\
\hline LC. Conductas positivas & 9.07 & 3.09 & 8.81 & 3.30 & 10.67 & 2.48 & $10.47 * * *$ \\
\hline LC. Conductas negativas & 5.53 & 4.23 & 4.63 & 3.89 & 3.75 & 3.33 & $4.71 * *$ \\
\hline LC. Índice de bullying & 0.28 & 0.67 & 0.23 & 0.69 & 0.10 & 0.46 & 2.04 \\
\hline LC. Índice general de agresión & 0.94 & 1.48 & 0.77 & 1.27 & 0.40 & 1.08 & $4.17 *$ \\
\hline AD Conducta antisocial-delictiva & 7.91 & 7.75 & 8.64 & 7.58 & 8.49 & 5.60 & 0.22 \\
\hline CTI. Pensamiento constructivo global & 87.95 & 10.20 & 93.34 & 9.99 & 96.41 & 11.47 & $13.30 * * *$ \\
\hline CTI. Emotividad & 76.32 & 9.33 & 80.63 & 10.09 & 81.39 & 12.16 & $5.09 * *$ \\
\hline CTI. Eficacia & 44.92 & 7.16 & 46.27 & 6.66 & 49.35 & 8.22 & $8.07 * * *$ \\
\hline CTI. Pensamiento supersticioso & 21.49 & 5.09 & 20.00 & 4.30 & 18.10 & 3.98 & $12.63 * * *$ \\
\hline CTI. Rigidez & 50.41 & 6.85 & 46.07 & 7.44 & 44.40 & 7.77 & $14.21 * * *$ \\
\hline CTI. Pensamiento esotérico & 34.77 & 7.95 & 32.56 & 7.42 & 28.41 & 6.92 & $16.63 * * *$ \\
\hline CTI. Ilusión & 50.07 & 8.77 & 49.30 & 6.44 & 49.48 & 7.09 & 0.21 \\
\hline
\end{tabular}

Como se puede observar en la tabla 4 , en recepción de conductas sociales positivas hay diferencias en función del nivel socio-económico-cultural, con puntuaciones superiores a medida que aumenta el nivel; no obstante la prueba de Tukey no evidenció diferencias entre el nivel bajo y medio. Aunque no se han encontrado diferencias en el índice de bullying, si se han hallado diferencias en el índice general de agresión, aunque únicamente entre el nivel bajo y alto, con puntuaciones superiores en el bajo, ya que la prueba de Tukey no confirmó diferencias entre el nivel bajo y medio, ni entre el nivel medio y alto. En lo que se refiere a la realización de conductas antisociales-delictivas no se han hallado diferencias significativas. En inteligencia emocional, excepto en la variable ilusión donde no se han encontrado diferencias entre los distintos niveles socio-económico-culturales, en el resto de las variables hay diferencias significativas, aumentando la puntuación en inteligencia emocional y las dimensiones asociadas a ésta, a medida que aumenta el estatus. No obstante, las pruebas de Tukey no muestran diferencias en pensamiento constructivo global, en emotividad, y en rigidez entre el status medio y alto; ni tampoco hay diferencias en eficacia, pensamiento supersticioso y pensamiento esotérico entre los estatus bajo y medio.

\section{DISCUSION}

El estudio tuvo como objetivo principal analizar las relaciones existentes entre ser víctima de acoso escolar y ser agresor con inteligencia emocional. En primer lugar, los resultados obtenidos han puesto de relieve que los adolescentes que recibían muchas conductas sociales positivas, tenían alto pensamiento constructivo global o inteligencia emocional (una estructura de pensamiento flexible, capacidad para adaptar su forma de pensar a las diferentes situaciones, nivel adecuado de aceptación de sí y de los demás, 
capacidad para establecer relaciones gratificantes, tendencia a conceder a otros el beneficio de la duda...), alto nivel de pensamiento positivo (capacidad de enfatizar el aspecto positivo de la situaciones y convertir tareas desagradables en lo menos penosas posibles), alto nivel de responsabilidad (capacidad para planificar objetivos y realizar acciones para llevarlos a cabo), poca suspicacia (bajo nivel de desconfianza), pocas creencias irracionales (bajo nivel de creencia en supersticiones convencionales, astrología...), alto nivel de ilusión (visión simplista de la vida, tendencia a evitar realidades desagradables), y de ingenuidad (creencia que considera que todo el mundo tiene buen corazón). Estos resultados ratifican la hipótesis 1 y apuntan en la misma dirección que los hallados por Lopes et al. (2003) y Ciarrochi et al. (2001).

En segundo lugar, los adolescentes que habían sufrido muchas conductas de intimidación o bullying y los que tenían alto índice general de agresión, también tuvieron bajo nivel de pensamiento constructivo global o inteligencia emocional (una estructura de pensamiento poco flexible, baja capacidad para adaptar su forma de pensar a las diferentes situaciones, nivel bajo de aceptación de sí y de los demás, baja capacidad para establecer relaciones gratificantes, poca tendencia a conceder a otros el beneficio de la duda), baja emotividad (poca capacidad de enfrentarse a las situaciones estresantes, baja autoaceptación, excesiva sensibilidad a las críticas o rechazos, tendencia a recordar adversidades pasadas, a tener pensamientos negativos y al estrés), baja autoestima (actitud poco favorable hacia sí mismo), baja tolerancia a la frustración (tendencia a sobrestimar las experiencias desfavorables), baja eficacia (poco optimismo, entusiasmo, energía... es decir, pocas actitudes que contribuyen a actuar con eficacia), y poca actividad (baja capacidad para aceptar desafíos y no abandonar ante el fracaso). Los datos confirman la hipótesis 2 y ratifican los resultados de los estudios de Hoover et al. (1992), y también los estudios que han evidenciado baja autoestima en las víctimas (Eslea et al., 2004; De la Torre et al., 2008; Estévez et al., 2006; Hanish y Guerra, 2004; Mynard y Joseph, 1997; Nansel et al., 2001; O’Moore, 1997; Olweus, 1993; Piñuel y Oñate, 2006; Slee y Rigby, 1993; Van Der Meulen et al., 2003).

En tercer lugar, los adolescentes que tenían un nivel alto de conductas antisociales-delictivas mostraban bajo nivel de pensamiento constructivo global o inteligencia emocional, bajo nivel de eficacia, de actividad, de responsabilidad, de extremismo, de ilusión, de pensamiento estereotipado y de tolerancia, lo que confirma la hipótesis 3. Estos resultados confirman los obtenidos por Caspi et al. (1994), Contreras y García (2008), Liau et al. (2003), Zimmerman (2005) y Zuckerman (1994), y principalmente en lo referente a deficiencias de autocontrol y empatía, los estudios de Avilés y Monjas (2005), Díaz-Aguado (2006), Henley y Long (1999), Hernández et al. (2002) y Stassen (2007).

Finalmente, los hallazgos del estudio confirman diferencias significativas en IE en función del estatus socio-económico-cultural, con mayores puntuaciones en IE a 
medida que aumenta el estatus, sin embargo, no se hallaron diferencias en el índice de bullying ni en la realización de conductas antisociales-delictivas, por lo que la hipótesis 4 se confirma parcialmente.

Los resultados han evidenciado que tanto las víctimas de acoso escolar como los agresores tienen bajo nivel de IE. Estos hallazgos tienen implicaciones prácticas y permiten sugerir la necesidad de implementar programas de intervención en contextos educativos que estimulen la IE (autoestima, tolerancia a la frustración, afrontamiento de situaciones estresantes, optimismo, afrontamiento de desafíos con orientación de éxito, responsabilidad...), ya que tendrán implicaciones positivas de cara a disminuir el número de niños y adolescentes que sufrirán situaciones de violencia o acoso por parte de los iguales, así como el número de niños y adolescentes que realizarán este tipo de conductas tan nocivas tanto para las víctimas como para los agresores. Esta necesidad de fomentar la educación emocional también ha sido puesta de relieve en estudios recientes (Peña y Canga, 2009)

\section{Agradecimientos}

Estudio financiado por el Departamento de Educación, Universidades e Investigación del Gobierno Vasco (GIC07/57).

\section{REFERENCIAS}

Arora, C.M.J. (1987). My Life in School Checklist. Transcrito por S. Sharp, C. M.J. Arora, P.K. Smith y I.Whitney (1994). How to measure bullying in your school. En S. Sharp y P.K. Smith (Eds.), Tackling bullying in your school (pp. 7-21). London: Routledge.

Avilés, J.M. y Monjas, I. (2005). Estudio de la incidencia de la intimidación y el maltrato entre iguales en la educación secundaria obligatoria mediante el cuestionario CIME. Anales de Psicología, 21(1), 27-41.

Batsche, G.M. y Knoff, H.M. (1994). Bullies and their victims: Understanding a pervasive problem in the schools. School Psychology Review, 23(2), 165-174.

Baumeister, R.F. (2001). Raíces de la violencia. Investigación y Ciencia, 297, 76-81.

Cammack-Barry, T. (2005). Long-term impact of elementary school bullying victimization on adolescents. Dissertation Abstracts International: Section B: The Sciences and Engineering, 65(9-B), 4819.

Caspi, A., Moffitt, T.E., Silva P.A., Stouthamer-Loeber, M., Krueger, R.F. y Schmutte, P.S. (1994). Are some people crime-prone? Replications of the personality-crime relationship across countries, genders, races, and methods. Criminology, 32, 163-95.

Ciarrochi, J., Chan, A. y Bajgar, J. (2001). Measuring emotional intelligence in adolescents. Personality and Individual Differences, 31(7), 1105-1119.

Contreras, A. y García, J.A. (2008). Componentes cognitivos que favorecen la adaptación social en la infancia: prevención del bullying desde la educación infantil. International Journal of Psychology and Psychological Therapy, 8(2), 217-226.

De la Torre, M.J., García, M.C., Carpio, M.V. y Casanova, P.F. (2008). Relaciones entre la vida escolar y autoconcepto multidimensional en Educación Secundaria Obligatoria. European Journal of Education and Psychology, 1(2), 57-70. 
Díaz-Aguado, M.J. (2004). Prevención de la violencia y lucha contra la exclusión desde la adolescencia. La violencia entre iguales en la escuela y en el ocio. Volumen 2: Programa de intervención y estudio experimental. Madrid: Instituto de la Juventud. Ministerio de Trabajo y Asuntos Sociales.

Díaz-Aguado, M.J. (2006). Sexismo, violencia de género y acoso escolar. Propuestas para una prevención integral de la violencia. Revista de Estudios de Juventud, 73, 38-58.

Epstein, N. (2001). CTI. Inventario de pensamiento constructivo. Una medida de la Inteligencia Emocional. Madrid: TEA.

Eslea, M., Menesini, E., Morita, Y., O’Moore, M., Mora-Merchán, J.A., Pereira, B., Smith, P.K. y Wenxin, Z. (2004). Friendship and loneliness among bullies and victims: Data from seven countries. Aggressive Behavior, 30, 71-83.

Esteve, J.M., Merino, D. y Cantos, B. (2001). La escolarización de los niños inmigrantes en el Campo de Gibraltar. Proyecto de Investigación financiado con fondos FEDER. Trabajo de investigación no publicado. Universidad de Málaga.

Estévez, E., Herrero, J., Martínez, B. y Musitu, G. (2006). Aggressive and non-aggressive rejected students: An analysis of their differences. Psychology in The Schools, 43, 387-400.

Estévez, E., Martínez, B. y Musitu, G. (2006). La autoestima en adolescentes agresores y víctimas en la escuela: la perspectiva multidimensional. Intervención Psicosocial, 15(2), 223-233.

Garaigordobil, M. (2009). Evaluación del programa "Dando pasos hacia la paz - Bakerako urratsak". Vitoria-Gasteiz: Servicio de Publicaciones del Gobierno Vasco.

Garaigordobil, M. y Oñederra, J.A. (2010). La violencia entre iguales: Revisión teórica y estrategias de intervención. Madrid: Pirámide.

Goleman, D. (1996). Inteligencia emocional. Barcelona: Kairós.

Hanish, L. y Guerra, N.G. (2004). Aggressive victims, passive victims, and bullies: developmental continuity or developmental change? Merrill Palmer Quarterly, 50(1), 17-38.

Henley, M. y Long, N.J. (1999). Teaching emotional intelligence to impulsive- aggressive youth. Reclaiming Children and youth, 7(4), 224-229.

Hernández, T., Sarabia, B. y Casares, E. (2002). Incidencia de variables contextuales en la violencia "bullying" en el recinto escolar. Psicothema, 14(supl.), 50-62.

Hoover, J.H., Oliver, R. y Hazler, R.J. (1992). Bullying: Perceptions of adolescent victims in the Midwestern USA. School Psychology International, 13(1), 5-16.

León, B. (2009). Salud mental en las aulas. Revista de Estudios de Juventud, 84, 66-83.

Liau, A.K., Liau, A.W.L., Teoh, G.B.S. y Liau, M.T.L. (2003). The Case for Emotional Literacy: the influence of emotional intelligence on problem behaviours in Malaysian secondary school students. Journal of Moral Education, 32(1), 51-66.

Lopes, P.N., Salovey, P. y Straus, R. (2003). Emotional intelligence, personality and the perceived quality of social relationships. Personality and Individual Differences, 35(3), 641-658.

Martorell, M., González, R., Rasal, P. y Estellés, R. (2009). Convivencia e inteligencia emocional en niños en edad escolar. European Journal of Education and Psychology, 2(1), 69-78.

Mynard, H. y Joseph, S. (1997). Bully/victim problems and their association with Eysenck's personality dimensions in 8 to 13 year-olds. British Journal of Educational Psychology, $67,51-54$.

Nansel, T.R., Overpeck, M., Pilla, R.S., June, W., Simons-Morton, B. y Scheidt, P. (2001). Bullying Behaviors Among U.S. Youth: Prevalence and Association with Psychosocial Adjustment. Journal of the American Medical Association, 285(16), 2094-2100.

O’Moore, A.M. (1997). Self-concept and Bullying Behaviour among School children and adolescent. Abstract, $5^{\text {th }}$. European Congress of Psychology. Dublin, 100.

Olweus, D. (1993). Victimization by peers: antecedents and long-term outcomes. En K.H. Rubin y J.B. Asendorf (Eds.), Social withdrawal, inhibition, and shyness in childhood (pp. 315- 
341). Hillsdale, N.J.: Lawrence Erlbaum.

Parkhurst, J.T. y Asher, S.R. (1992). Peer rejection in middle school: subgroups differences in behavior, lonelines, and interpersonal concerns. Developmental Psychology, 28, 231241.

Pelegrín, A. y Garcés de Los Fayos, E.J. (2008). Variables contextuales y personales que inciden en el comportamiento violento del niño. European Journal of Education and Psychology, 1(1), 5-20.

Peña, A.M. y Canga, M.C. (2009). La educación emocional en el contexto escolar con alumnado de distintos países. European Journal of Education and Psychology, 2(3), 199-210.

Piñuel, I. y Oñate, A. (2006). Estudio Cisneros X: Violencia y Acoso escolar en España. Instituto de Innovación Educativa y Desarrollo Directivo (IEDDI). http://www.fapacne.com/ publicaciones/acoso-escolar/acoso-escolar.pdf

Rubin, M.M. (1999). Emotional intelligence and its role in mitigating aggression: A correlational study of the relationship between emotional intelligence and aggression in urban adolescents. Tesis doctoral no publicada. Immaculata College, Inmaculata, PA, Estados Unidos.

Seisdedos, N. (1995). AD. Cuestionario de conductas antisociales y delictivas. Madrid: TEA. (trabajo original publicado en 1988)

Slee, P.T. y Rigby, K. (1993). The relationship of Eysenck's personality factors and self-steem to bully/victim behaviour in Australian school boys. Personality and Individual Differences, 14, 371-373.

Stassen, K. (2007). Update on bullying at school: science forgotten? Developmental Review, 27, 90-126.

Van Der Meulen, K., Soriano, L., Granizo, L., Del Barrio, C., Korn, S., y Scháfer, M. (2003). Recordando el maltrato entre iguales en la escuela: consecuencias e influencia en la actuación del profesorado. Infancia y Aprendizaje, 26(1), 49-62.

Zimmerman, F.J. (2005). Early cognitive stimulation, emotional support, and television watching as predictors of subsequent bullying among grade-school children. Archives of Pediatrics y Adolescent Medicine, 159(4), 384-388.

Zuckerman, M. (1994). Behavioral expressions and biosocial bases of sensation seeking. Cambridge: Cambridge University Press.

Recibido: 2 de enero de 2010

Recepción Modificaciones: 12 de enero de 2010

Aceptado: 22 de enero de 2010 